\title{
Noninvasive Ventilation for Acute Hypercapnic Respiratory Failure: Intubation Rate in an Experienced Unit
}

\author{
Damien Contou MD, Chiara Fragnoli MD, Ana Córdoba-Izquierdo MD, \\ Florence Boissier MD, Christian Brun-Buisson MD, and Arnaud W Thille MD PhD
}

\begin{abstract}
BACKGROUND: Failure of noninvasive ventilation (NIV) is common in patients with COPD admitted to the ICU for acute hypercapnic respiratory failure (AHRF). We aimed to assess the rate of NIV failure and to identify early predictors of intubation under NIV in patients admitted for AHRF of all origins in an experienced unit. METHODS: This was an observational cohort study using data prospectively collected over a 3-year period after the implementation of a nurse-driven NIV protocol in a 24-bed medical ICU of a French university hospital. RESULTS: Among 242 subjects receiving NIV for AHRF $\left(\mathrm{P}_{\mathrm{aCO}_{2}}>45 \mathrm{~mm} \mathrm{Hg}\right), 67$ had cardiogenic pulmonary edema (CPE), 146 had acute-on-chronic respiratory failure (AOCRF) (including 99 subjects with COPD and 47 with other chronic respiratory diseases), and 29 had non-AOCRF (mostly pneumonia). Overall, the rates of intubation and ICU mortality were respectively $15 \%$ and $5 \%$. The intubation rates were $4 \%$ in CPE, $15 \%$ in AOCRF, and 38\% in non-AOCRF $(P<.001)$. After adjustment, non-AOCRF was independently associated with NIV failure, as well as acidosis $(\mathrm{pH}<7.30)$ and severe hypoxemia $\left(\mathrm{P}_{\mathrm{aO}_{2}} / \mathrm{F}_{\mathrm{IO}_{2}} \leq 200 \mathrm{~mm} \mathrm{Hg}\right)$ after 1 hour of NIV initiation, whereas altered consciousness on admission and ventilatory settings had no influence on outcome. CONCLUSIONS: With a nurse-driven NIV protocol, the intubation rate was reduced to $15 \%$ in patients receiving NIV for AHRF, with a mortality rate of only 5\%. Whereas the risk of NIV failure is associated with hypoxemia and acidosis after initiation of NIV, it is also markedly influenced by the presence or absence of an underlying chronic respiratory disease. Key words: noninvasive ventilation; acute respiratory failure; acute-on-chronic respiratory failure; cardiogenic pulmonary edema; COPD; hypercapnic coma; endotracheal intubation. [Respir Care 2013;58(12):2045-2052. (C) 2013 Daedalus Enterprises]
\end{abstract}

\section{Introduction}

Noninvasive ventilation (NIV) reduces the rates of intubation and mortality in patients with severe exacerbation of $\mathrm{COPD}^{1,2}$ or cardiogenic pulmonary edema $(\mathrm{CPE}) .^{3} \mathrm{In}$ our ICU, NIV has been used since the late 1980s, and was shown by Brochard et $\mathrm{al}^{4}$ to be beneficial in patients ad-

The authors are affiliated with Réanimation Médicale, Hôpitaux Universitaires Henri Mondor, Assistance Publique Hôpitaux de Paris, Créteil, France. Dr Thille is also affiliated with Institut National de la Santé et de la Recherche Médicale U955, Créteil, France, and with the Réanimation Médicale, Centre Hospitalier de l'Université de Poitiers, Poitiers, France.

Dr Contou presented a version of this paper at the 2012 meeting of the European Society of Intensive Care Medicine, held October 13-17, 2012, in Lisbon, Portugal. mitted with exacerbation of COPD. A subsequent prospective randomized study demonstrated that NIV was associated with reduced rates of endotracheal intubation and mortality in these patients. ${ }^{5}$

Several large surveys show that the use of NIV has become widespread in treatment of severe exacerbation of

\footnotetext{
Supplementary material related to this paper is available at http:// www.rcjournal.com.

The authors have disclosed no conflicts of interest.

Correspondence: Damien Contou MD, Réanimation Médicale, Hôpitaux Universitaires Henri Mondor, 51 Avenue du Maréchal de Lattre de Tassigny, 94010 Créteil, France. E-mail: contou@club-internet.fr.
}

DOI: $10.4187 /$ respcare.02456 
COPD in Europe and in the United States. ${ }^{6-8}$ Despite the increasing experience with this technique, the rate of NIV failure remains high, between $20 \%$ and $30 \%$ in COPD patients admitted to ICUs. ${ }^{2,5,9,10}$ NIV may also be used as first-line management of non-COPD patients having acute hypercapnic respiratory failure (AHRF), but the rate of NIV failure and intubation can exceed $30-40 \%$ in this group. ${ }^{8,11,12}$ In COPD patients the severity of hypercapnia and/or acidosis after initiation of NIV is a major predictor of NIV failure. ${ }^{11,13-15}$ However, no study has evaluated the impact of the NIV ventilatory settings and respiratory parameters under NIV on outcome, and little information is available on hypercapnic non-COPD patients treated with NIV.

The aims of this study were to assess the rate of NIV failure in patients admitted for AHRF, whatever the cause, in an experienced unit, and to identify early predictors of intubation under NIV. Some of the results of this study have been previously reported in the form of an abstract at the 2012 meeting of the European Society of Intensive Care Medicine in Lisbon, Portugal. ${ }^{16}$

\section{Methods}

This observational cohort study was conducted in our 24-bed medical ICU at Hôpitaux Universitaires Henri Mondor in Créteil, France. The institutional review board of the French Society for Respiratory Medicine approved this noninterventional study and waived the need for informed consent.

\section{Subjects}

We prospectively included all consecutive patients admitted during a 3-year period (June 2008 to June 2011) and who received NIV as initial ventilatory support for AHRF. AHRF was defined as recent dyspnea with sternocleidomastoid muscle activation and a breathing frequency $>25$ breaths/min and/or an arterial $\mathrm{pH}<7.35$, with a $\mathrm{P}_{\mathrm{aCO}_{2}}$ above $45 \mathrm{~mm} \mathrm{Hg}$. We excluded patients who were intubated before ICU admission or intubated upon ICU admission without prior NIV, and patients for whom NIV was used with a do-not-intubate order.

\section{NIV Protocol and Definitions}

The study was conducted after the implementation, in June 2008, of a nurse-driven NIV protocol that included prospective daily collection of clinical data and ventilatory parameters on a specific NIV monitoring form. When the NIV form was unavailable or incomplete, data were retrieved from the subject's records.

All stages of the protocol had been developed within a multidisciplinary working group including ICU physicians,

\section{QUICK LOOK}

\section{Current knowledge}

Failure of noninvasive ventilation (NIV) is common in patients with COPD admitted to the ICU for acute hypercapnic respiratory failure. In selected patients the commonly reported failure rate is approximately $30 \%$.

\section{What this paper contributes to our knowledge}

With a nurse-driven NIV protocol, the intubation rate was reduced to $15 \%$ in patients receiving NIV for acute hypercapnic respiratory failure, with an accompanying mortality rate of only $5 \%$. The risk of NIV failure is associated with hypoxemia and acidosis after initiation of NIV, and is also markedly influenced by the presence or absence of an underlying chronic respiratory disease. Altered consciousness at admission and ventilator settings had no influence on outcome.

nurses, and respiratory therapists. A daily NIV prescription by the physician indicated the duration of NIV sessions and targeted expiratory tidal volume (around $6-8 \mathrm{~mL} /$ $\mathrm{kg})$ and $\mathrm{S}_{\mathrm{pO}_{2}}(88-92 \%$ in subjects with acute-on-chronic respiratory failure [AOCRF], and $\geq 94 \%$ in other subjects). Given that respiratory therapists are not present every day and all day long in our unit, the protocol aimed at empowering nurses to adjust the ventilatory settings and to improve the subject's tolerance to NIV. Nurses are not as highly skilled in mechanical ventilation as respiratory therapists in the United States can be, and were not involved in the decision to intubate. The first objective was to reach the targeted expiratory tidal volume and $\mathrm{S}_{\mathrm{pO}_{2}}$, and to improve the subject's tolerance to NIV following a simple decision algorithm (see the protocol and algorithm used in the supplementary material at http:// www.rcjournal.com).

Pressure-support ventilation was started, using a pressure-support level of $8 \mathrm{~cm} \mathrm{H}_{2} \mathrm{O}$, a PEEP level of $0 \mathrm{~cm} \mathrm{H}_{2} \mathrm{O}$, an inspiratory trigger of $3 \mathrm{~L} / \mathrm{min}$, and a maximal inspiratory time of 1 second. The nurses then adjusted the ventilatory parameters, including pressure-support level and $\mathrm{F}_{\mathrm{IO}_{2}}$, according to the protocol. Pressure-support level was gradually increased by $2 \mathrm{~cm} \mathrm{H}_{2} \mathrm{O}$ steps to reach the target expiratory tidal volume, and PEEP level was then adjusted as prescribed. $\mathrm{F}_{\mathrm{IO}_{2}}$ was gradually adjusted by $5 \%$ steps to reach the targeted $\mathrm{S}_{\mathrm{pO}_{2}}$. NIV was applied intermittently for periods of at least 2 hours, with a minimal duration of 6 hours per day, or continuously in case of hypercapnic coma, and was maintained until signs of respiratory distress improved. An algorithm was used by nurses in case of leaks, which involved repositioning of the mask, then reducing the PEEP by $2 \mathrm{~cm} \mathrm{H}_{2} \mathrm{O}$, then 


\section{Noninvasive Ventilation for Acute Hypercapnic Respiratory Failure}

reducing the pressure-support level by steps of $2 \mathrm{~cm} \mathrm{H}_{2} \mathrm{O}$ until the minimal expiratory volume was reached, and then changing the mask interface. Continuous mandatory ventilation could be used transiently in subjects with hypercapnic coma and triggering insufficient tidal volume despite high pressure support levels.

A mobile cart containing all types and sizes of interfaces was available at the bedside during initiation of NIV. NIV was performed via a non-vented oronasal mask (FreeMotion RT041, Fisher \& Paykel, Auckland, New Zealand, or Ultra Mirage, ResMed, San Diego, California), with an ICU ventilator using a dedicated NIV mode (Evita XL, Dräger, Lübeck, Germany, or Engström CareStation, GE Healthcare, Little Chalfont, Buckinghamshire, United Kingdom), equipped with a heated humidifier (MR850, Fisher \& Paykel, Auckland, New Zealand).

The following criteria were used for endotracheal intubation: hypercapnic coma with inability to deliver tidal volume, loss of consciousness or persistent hypercapnic coma under NIV, psychomotor agitation making nursing care impossible and requiring sedation, pronounced worsening in signs of respiratory distress with a breathing frequency above 40 breaths/min under NIV, $\mathrm{S}_{\mathrm{pO}_{2}}$ remaining below $90 \%$ despite $\mathrm{F}_{\mathrm{IO}_{2}} 1.0$, and persistent hypotension despite fluid resuscitation requiring vasopressors. Worsening respiratory acidosis or absolute values of $\mathrm{pH} / \mathrm{P}_{\mathrm{CO}_{2}}$ were not used as criteria for intubation in the absence of other signs cited above.

\section{Data Collection}

From the NIV monitoring forms we analyzed the number and duration of NIV sessions, ventilator settings (pressure support level, PEEP, $\mathrm{F}_{\mathrm{IO}_{2}}$ ), ventilatory parameters $\left(\mathrm{S}_{\mathrm{pO}_{2}}\right.$, breathing frequency, expiratory tidal volume), level of consciousness (assessed using the Richmond AgitationSedation Scale), ${ }^{17}$ NIV tolerance (scored from 0 for "poor" to 3 for "excellent"), amount of leaks (scored from 0 for "no leaks" to 3 for "major"), and hemodynamic parameters (heart rate, blood pressure). Poor tolerance was considered as a score of 0 or 1 , and major leaks as a score of 2 or 3. Altered consciousness was defined as a Richmond Agitation-Sedation Scale $<0$, and coma as a Glasgow coma score $\leq 8$. Blood gases were routinely measured 1 hour after initiation of NIV. Clinical data (breathing frequency, $\mathrm{S}_{\mathrm{pO}_{2}}$, blood pressure, heart rate, Glasgow coma score) and blood gases at admission before NIV initiation were retrospectively collected from the medical chart. An independent pulmonologist classified subjects according to the underlying cause of AHRF into one of 3 subgroups: CPE, AOCRF (including subjects having chronic respiratory failure associated with COPD or with other, nonCOPD causes), and non-AOCRF, which included subjects without underlying chronic respiratory disease.

\section{Statistical Analysis}

Data are expressed as mean $\pm \mathrm{SD}$, median and IQR, or number and percent (for dichotomous variables). Qualitative data were compared using the chi-square test, and quantitative data using the unpaired Student $t$ test or Kruskal Wallis test.

To evaluate independent factors associated with NIV failure at admission, univariate risk factors with a $P$ value $<.10$ were examined using backward stepwise logistic regression analysis. Among related significant univariate factors, only the most clinically relevant were entered into the regression model in order to minimize the effect of collinearity. We therefore included the cause of AHRF, tachypnea, and altered consciousness at admission, and hypoxemia and respiratory acidosis after NIV initiation, whereas Simplified Acute Physiology Score II was not entered into the model. We considered 2-tailed $P$ values $<.05$ as significant. The statistical analysis was performed using statistics software (Stata 10.1, StataCorp, College Station, Texas).

\section{Results}

\section{Subjects}

Over the 3-year period, 242 subjects received NIV for AHRF, including 67 with cardiogenic pulmonary edema (CPE), 146 with AOCRF, and 29 with non-AOCRF (Fig. 1). Prospective data on NIV ventilatory parameters was available for 83\% (201/242). Among the 47 subjects with nonCOPD AOCRF, 30 had obesity and/or obstructive sleep apnea syndrome (median body mass index $38 \mathrm{~kg} / \mathrm{m}^{2}$ ), while others had bronchiectasis $(n=4)$, permanent ventilatory impairment due to asthma $(n=4)$, pulmonary cancer $(n=2)$, chest-wall disease $(n=3)$, myopathy $(n=2)$, and myasthenia gravis $(n=2)$. Among the 29 subjects having non-AOCRF and hypercapnia, 24 had pneumonia (including 8 subjects with clinical criteria for ARDS), and 5 had drug intoxication. The subjects' characteristics at admission and their outcomes in ICU are reported in Table 1. Overall, 31 subjects were comatose either at admission $(n=15)$ or during the first 24 hours $(n=16)$.

\section{Rates of NIV Failure and ICU Mortality}

The overall rates of intubation and mortality were respectively $15 \%(36 / 242)$ and $5 \%(13 / 242)$. The intubation rates were 4\% (3/67) in CPE, 15\% (22/146) in AOCRFwith an identical rate in COPD and non-COPD subjectsand 38\% (11/29) in non-AOCRF $(P<.001)$ (Fig. 2$)$. The corresponding ICU mortality rates were $3 \%(2 / 67), 5 \%$ (7/146), and 14\% (4/29) $(P=.08)$ (Fig. 3). The intubation rates were $11 \%(13 / 115)$ in subjects having a $\mathrm{pH}$ on ad- 


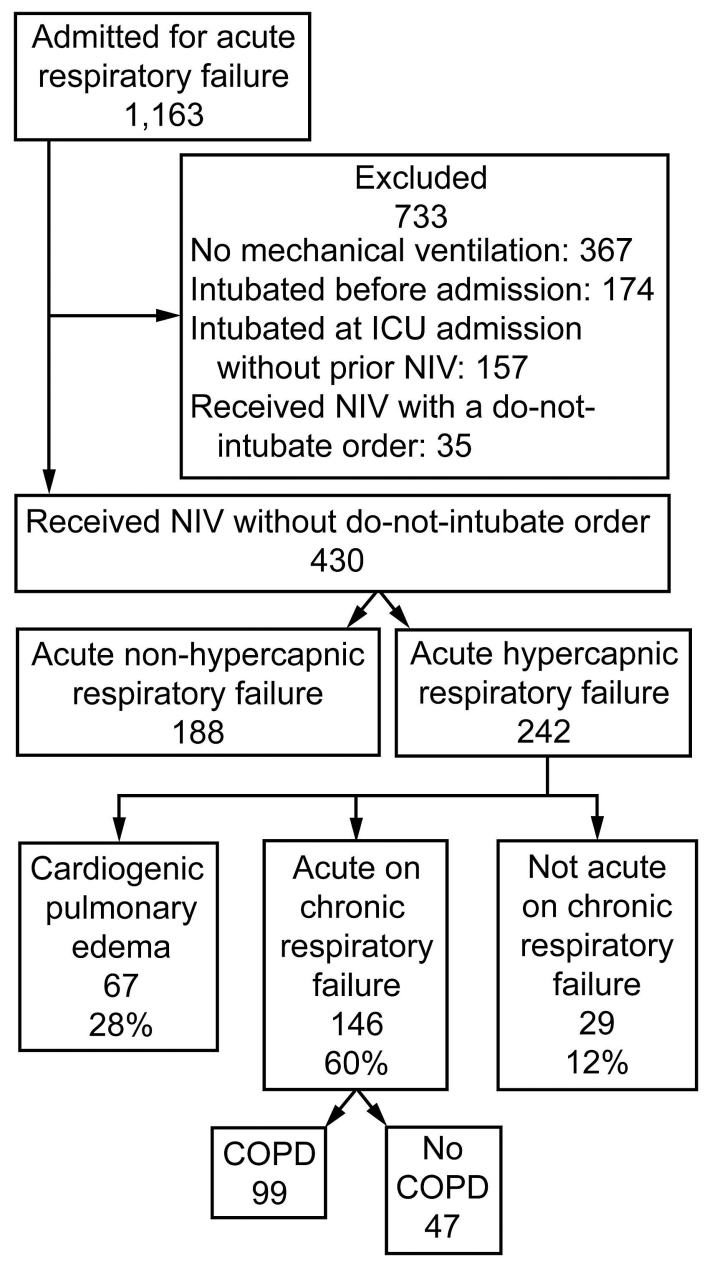

Fig. 1. Flow chart. NIV = noninvasive ventilation.

mission $\geq 7.30$ and $18 \%(23 / 128)$ in those having a $\mathrm{pH}<7.30(P=.15)$. The in-ICU mortality rate of intubated subjects was $36 \%(13 / 36)$.

\section{Factors Associated With NIV Failure}

Among variables recorded on ICU admission, the etiology of AHRF and tachypnea ( $>30$ breaths/min) was independently associated with NIV failure (Table 2). NonAOCRF was an independent predictor of NIV failure, as compared to subjects with AOCRF and CPE.

Pressure-support level adjusted by nurses was significantly greater 1 hour after NIV initiation than at NIV initiation $\left(9.5 \pm 3.0 \mathrm{~cm} \mathrm{H}_{2} \mathrm{O}\right.$ vs $\left.9.2 \pm 2.6, P=.036\right)$, while tidal volume remained similar $(468 \pm 144 \mathrm{~mL}$ vs $465 \pm 135, P=.64)$. Although not significantly different, expiratory tidal volume 1 hour after NIV initiation tended to be lower in subjects who failed NIV, compared to those who succeeded in NIV.

Among variables recorded at one hour after NIV initiation, ventilatory settings and subject tolerance to NIV or amount of leaks had no influence on outcome, whereas severe hypoxemia $\left(\mathrm{P}_{\mathrm{aO}_{2}} / \mathrm{F}_{\mathrm{IO}_{2}} \leq 200 \mathrm{~mm} \mathrm{Hg}\right)$ and severe acidosis $(\mathrm{pH}<7.30)$ were independently associated with NIV failure.

After adjustment, altered consciousness at admission was not associated with NIV failure and only $23 \%(14 / 60)$ of subjects who had encephalopathy were intubated. Among the 31 comatose subjects, 15 (48\%) succeeded in NIV without need for endotracheal intubation.

\section{Discussion}

In hypercapnic patients receiving NIV as first-line ventilatory support for acute respiratory failure of various origins, we found that the overall rate of intubation was only $15 \%$. However, this rate differed markedly according to the underlying cause of acute respiratory failure, and reached 38\% in patients without chronic respiratory disease (non-AOCRF). Among patients with AOCRF, no difference was found between COPD and non-COPD patients.

\section{Rate of NIV Failure According to the Cause for Acute Hypercapnic Respiratory Failure}

The intubation rate of only $4 \%$ in subjects receiving NIV for CPE compares favorably with the $14 \%$ rate reported in a meta-analysis ${ }^{3}$ and the $18 \%$ rate reported in a survey from the United States. ${ }^{12}$ Some studies have even reported intubation rates exceeding $20 \%$ in the subset of hypercapnic patients. ${ }^{18}$ Our results are, however, consistent with those of Nava et al, ${ }^{19}$ who reported an intubation rate of only $6 \%$ in hypercapnic patients with CPE treated in an ICU having extensive experience with NIV.

The $15 \%$ intubation rate we recorded in subjects with AOCRF is also lower than the $20-30 \%$ rates usually reported in studies evaluating NIV in COPD patients. $., 8-10,12$ Plant et al reported an overall intubation rate of only $15 \%$ in patients receiving NIV in respiratory wards, but this rate reached $36 \%$ in patients with a $\mathrm{pH}<7.30,{ }^{20}$ whereas only $18 \%$ of our subjects with a $\mathrm{pH}<7.30$ needed intubation. A recent study reported a rate of NIV failure of only $11 \%$ in severe COPD patients admitted to a specialized respiratory ICU, ${ }^{21}$ with an ICU mortality rate of $8 \%$, which is close to the 5\% recorded in our study. In this large observational study, rates of NIV failure and mortality were significantly lower in patients with obesityhypoventilation syndrome than in those with COPD. ${ }^{21} \mathrm{We}$ found a similarly low risk of NIV failure (15\%) in subjects having COPD or another underlying chronic respiratory disease. Indeed, NIV has been successfully used in obese patients with severe obstructive sleep apnea syndrome ${ }^{21-23}$ or bronchiectasis, ${ }^{24}$ and may also be effective, despite mixed 


\section{Noninvasive Ventilation for Acute Hypercapnic Respiratory Failure}

Table 1. Characteristics and Outcomes of 242 Subjects Receiving Noninvasive Ventilation for Acute Hypercapnic Respiratory Failure of All Origins

\begin{tabular}{|c|c|c|c|c|c|}
\hline & $\begin{array}{l}\text { All AHRF } \\
n=242\end{array}$ & $\begin{array}{c}\text { CPE } \\
n=67\end{array}$ & $\begin{array}{l}\text { AOCRF } \\
n=146\end{array}$ & $\begin{array}{c}\text { Non-AOCRF } \\
n=29\end{array}$ & $P$ \\
\hline Age, y & $70 \pm 15$ & $76 \pm 11^{*}$ & $70 \pm 12$ & $56 \pm 21 *$ & $<.001$ \\
\hline Male, no. (\%) & $144(60)$ & $36(54)$ & $88(60)$ & $20(69)$ & .36 \\
\hline SAPS II & $35 \pm 14$ & $38 \pm 14 \dagger$ & $33 \pm 12 *$ & $37 \pm 18$ & $<.05$ \\
\hline Systolic arterial pressure, $\mathrm{mm} \mathrm{Hg}$ & $143 \pm 50$ & $165 \pm 81^{*}$ & $136 \pm 28^{*}$ & $130 \pm 25$ & $<.001$ \\
\hline Heart rate, beats/min & $99 \pm 21$ & $95 \pm 20$ & $100 \pm 21$ & $105 \pm 25$ & .11 \\
\hline Breathing frequency, cycles/min & $29 \pm 8$ & $32 \pm 7 \dagger$ & $28 \pm 8^{*}$ & $30 \pm 10$ & .01 \\
\hline Glasgow coma score & $14 \pm 2$ & $14 \pm 2$ & $14 \pm 2$ & $14 \pm 3$ & .06 \\
\hline $\mathrm{S}_{\mathrm{pO}_{2}}, \%$ & $91 \pm 10$ & $90 \pm 12$ & $91 \pm 9$ & $94 \pm 5$ & .17 \\
\hline $\mathrm{pH}$ & $7.28 \pm 0.09$ & $7.26 \pm 0.10 \dagger$ & $7.28 \pm 0.07$ & $7.31 \pm 0.10 \dagger$ & .02 \\
\hline $\mathrm{P}_{\mathrm{aCO}_{2}}, \mathrm{~mm} \mathrm{Hg}$ & $68 \pm 17$ & $62 \pm 15^{*}$ & $72 \pm 16^{*}$ & $59 \pm 16^{*}$ & $<.001$ \\
\hline $\mathrm{P}_{\mathrm{aO}_{2}}, \mathrm{~mm} \mathrm{Hg}$ & $99 \pm 65$ & $118 \pm 77^{*}$ & $91 \pm 62 \dagger$ & $96 \pm 38$ & $<.001$ \\
\hline Bicarbonate, $\mathrm{mmol} / \mathrm{L}$ & $33 \pm 7$ & $29 \pm 6^{*}$ & $35 \pm 6^{*}$ & $29 \pm 7 *$ & $<.001$ \\
\hline $\mathrm{P}_{\mathrm{aO}_{2}} / \mathrm{F}_{\mathrm{IO}_{2}}$ at NIV initiation, $\mathrm{mm} \mathrm{Hg}$ & $229 \pm 86$ & $235 \pm 88$ & $233 \pm 79$ & $199 \pm 107$ & .10 \\
\hline $\begin{array}{l}\text { Duration of NIV the first day, } \\
\text { median (IQR) h }\end{array}$ & $8(4-11)$ & $7(4-8) \dagger$ & $9(4-12)^{*}$ & $6(4-10)$ & .01 \\
\hline Total duration of NIV, median (IQR) d & $2(1-4)$ & $2(1-3)^{*}$ & $3(1-5)^{*}$ & $1(1-3)$ & $<.001$ \\
\hline Rate of NIV failure, no. (\%) & $36(14)$ & $3(4)^{*}$ & $22(15)$ & $11(38)^{*}$ & $<.001$ \\
\hline ICU stay, median (IQR) d & $6(4-9)$ & $4(3-6)^{*}$ & $7(5-9)$ & $8(6-14) \dagger$ & $<.001$ \\
\hline ICU mortality, no. (\%) & $13(5)$ & $2(3)$ & $7(5)$ & $4(14)$ & .09 \\
\hline $\begin{array}{l} \pm \text { Values are mean } \pm \text { SD. } \\
* P<.02 \text { as compared to all other subjects using the } \\
\dagger P<.05 \text { as compared to all other subjects using the } \\
\text { AHRF }=\text { acute hypercapnic respiratory failure } \\
\mathrm{CPE}=\text { cardiogenic pulmonary edema } \\
\text { AOCRF = acute-on-chronic respiratory failure } \\
\text { SAPS }=\text { Simplified Acute Physiology Score } \\
\text { NIV }=\text { noninvasive ventilation }\end{array}$ & & & & & \\
\hline
\end{tabular}

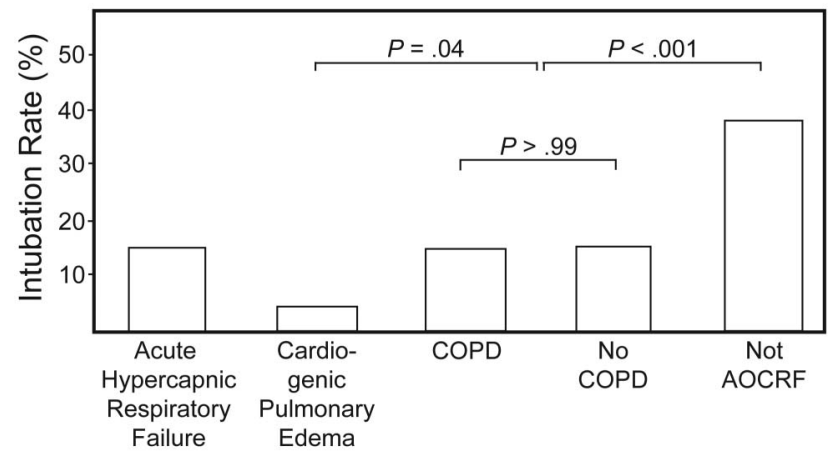

Fig. 2. Intubation rate in subjects receiving noninvasive ventilation for acute hypercapnic respiratory failure, overall and according to the reason for admission. AOCRF = acute-on-chronic respiratory failure.

results, in patients with restrictive pulmonary disease, ${ }^{25}$ myasthenia gravis, ${ }^{26}$ or neuromuscular disease. ${ }^{27}$ Identification of an underlying chronic respiratory disease other than COPD could be of major interest to better assess the risk of NIV failure in hypercapnic patients.

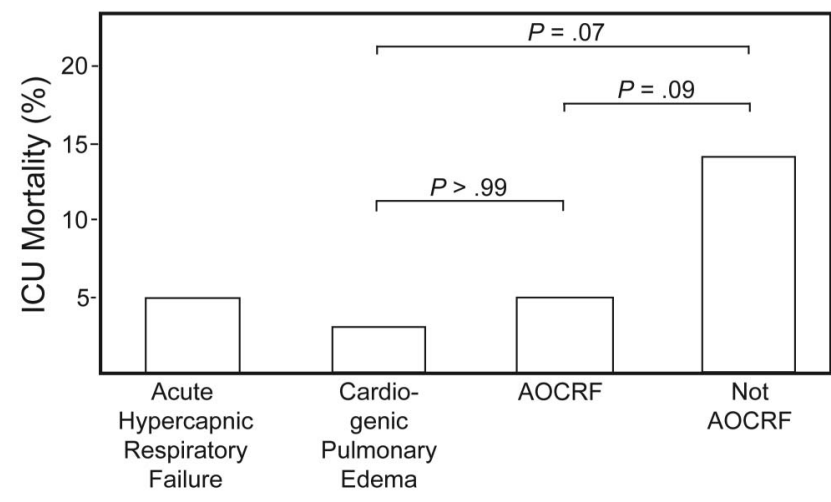

Fig. 3. ICU mortality in subjects receiving noninvasive ventilation for acute hypercapnic respiratory failure, overall and according to the reason for admission. AOCRF $=$ acute-on-chronic respiratory failure.

By contrast, we found a markedly higher rate (38\%) of NIV failure in hypercapnic subjects with non-AOCRF, mostly associated with pneumonia. High intubation rates of $38 \%^{12}$ or $47 \%^{11}$ have already been reported in non- 
Table 2. Predictors of Endotracheal Intubation in Subjects Admitted for Acute Hypercapnic Respiratory Failure Receiving Noninvasive Ventilation

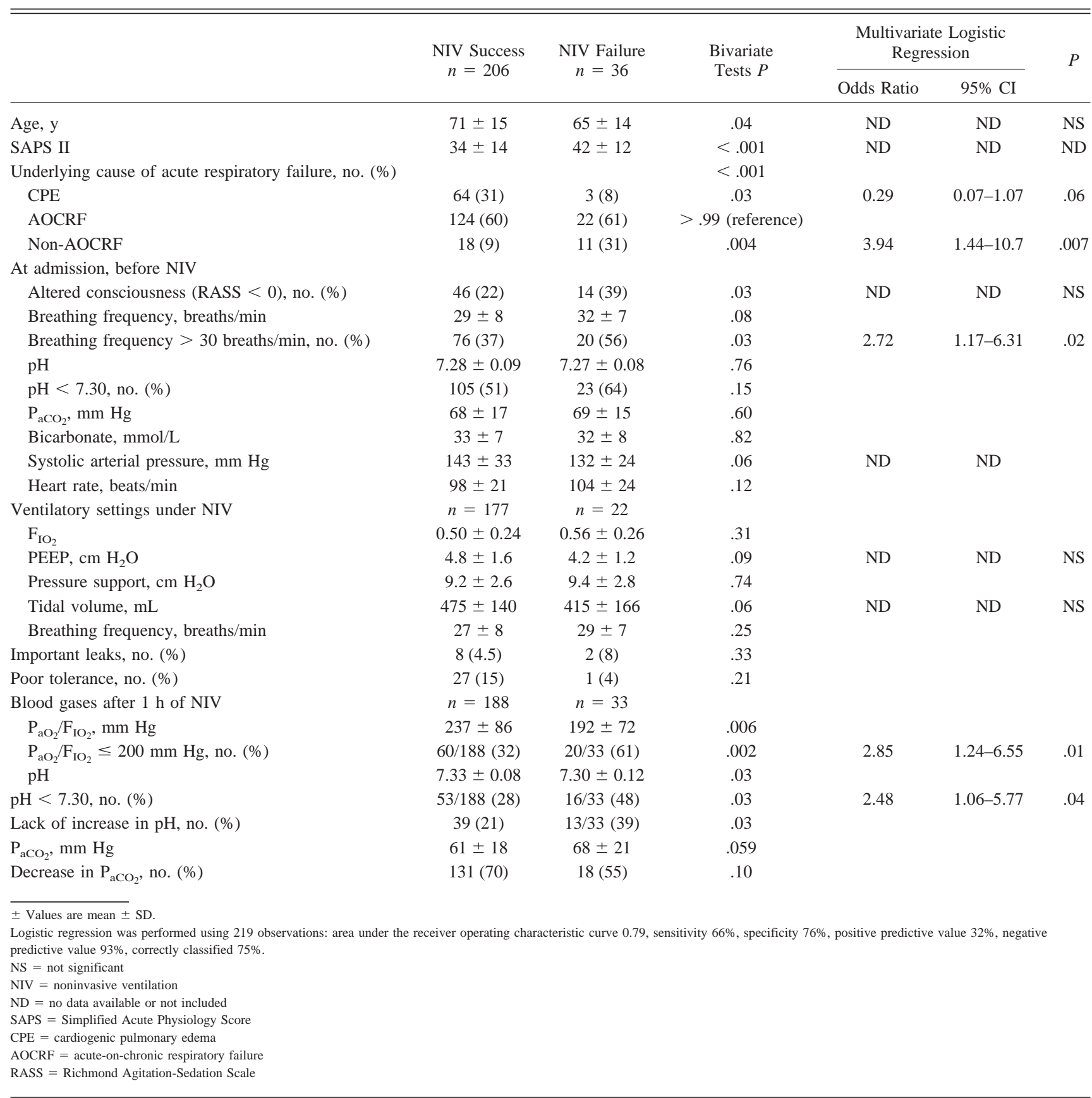

COPD patients receiving NIV for AHRF when including patients with and without underlying chronic respiratory disease. ${ }^{11,12}$ In patients having de novo acute hypoxemic (non-hypercapnic) respiratory failure and no chronic respiratory disease, even higher intubation rates of up to $60 \%$ have been reported. ${ }^{8,12}$ It is noteworthy that the intubation rate in our subgroup of patients having de novo acute hypercapnic respiratory failure was only $38 \%$, and that, although significantly less hypercapnic, they were not more hypoxemic than the others 2 subgroups.

\section{Predictive Factors for NIV Failure After NIV Initiation}

A higher severity score is usually associated with NIV failure in hypercapnic patients. ${ }^{11,15,28}$ However, using the Simplified Acute Physiology Score II is clinically impractical since this score is computed only at 24 hours after admission, therefore taking into account any potential complications of intubation in patients who failed NIV within the first 24 hours. 


\section{Noninvasive Ventilation for Acute Hypercapnic Respiratory Failure}

The severity of hypercapnia and/or respiratory acidosis after initiation of NIV is a well known predictor of NIV failure. ${ }^{11,13-15,29}$ Probably because we included AHRF of all origins, we also found that severe hypoxemia $\left(\mathrm{P}_{\mathrm{aO}_{2}} / \mathrm{F}_{\mathrm{IO}_{2}} \leq 200 \mathrm{~mm} \mathrm{Hg}\right)$ was an independent predictor of intubation in hypercapnic subjects.

By contrast, tolerance to NIV and amount of leaks had no impact on NIV failure. In a survey from 70 ICUs, poor NIV tolerance was a strong predictor of NIV failure. ${ }^{8} \mathrm{How}-$ ever, this study reported good NIV tolerance in only $27 \%$ of patients, and $57 \%$ had high levels of leaks. In our series, $86 \%$ of subjects had good NIV tolerance, and only $10 \%$ had high levels of leaks, probably due to our NIV protocol. Pressure-support level was significantly increased during the first hour of NIV, suggesting that the protocol was correctly applied by nurses. With such good NIV tolerance during the first hour of NIV, the ventilatory parameters or ventilator settings had no influence on outcome. However, the trend toward a lower tidal volume in patients who failed NIV, as compared to patients who avoided intubation, might suggest the need to increase the targeted tidal volume in patients at high risk of failure.

\section{Clinical Implications}

In a general ICU using protocolized care and monitoring of NIV by nurses, the overall rate of intubation in hypercapnic patients receiving NIV for acute respiratory failure could be maintained below $15 \%$. This rate can be used as an upper limit, both for COPD patients and for other patients having a chronic underlying respiratory disease. These results are probably due, first, to our NIV protocol optimizing the patient's tolerance to NIV, and, second, to our conservative intubation criteria, enabling continuation of NIV under close monitoring in some patients with altered consciousness. As expected, the rate of intubation was particularly high in patients with persistently or newly occurring severe altered consciousness. Nevertheless, $48 \%$ of our comatose patients achieved NIV without the need for intubation. Several studies have already shown that NIV could be successful in patients with hypercapnic coma. ${ }^{30,31}$ Moreover, it has been shown that NIV failure was not associated with an increased mortality rate in hypercapnic patients ${ }^{28}$; thus, delayed intubation in some patients likely did not worsen their outcome. In our study the ICU mortality rate for intubated patients was $36 \%$, which is in line with the $30-40 \%$ rate reported in large surveys. ${ }^{12,28}$ Our results also suggest that, similar to protocols for weaning from mechanical ventilation ${ }^{32}$ or sedation, ${ }^{33}$ which enabled reduction of the time to extubation, NIV protocols involving nurses and/or respiratory therapists might reduce the intubation rate.

\section{Limitations}

Our study was conducted in a single unit with longstanding experience in the practice of NIV, and therefore our results may not be applicable to other centers with less extensive experience. Experience and nurse-driven protocols may improve tolerance to NIV, and we report a poor tolerance rate of only $14 \%$ after 1 hour of NIV. Another limitation is the retrospective nature of the study. However, prospective data collection of ventilatory parameters under NIV was available for a vast majority of our subjects, and, because of the availability of computerized medical charts for all subjects, all those receiving NIV for AHRF could be analyzed.

\section{Conclusions}

While the rate of NIV failure is usually around 20 to $30 \%$ for acute hypercapnic respiratory failure, we found that the intubation rate could be maintained below $15 \%$ in a highly experienced unit, with an overall ICU mortality of only 5\%. Our study suggests that an NIV trial should be considered in all hypercapnic patients presenting with acute respiratory failure, even when the risk of failure is high because of coma, whether in patients with AOCRF or in patients without underlying respiratory disease. Interestingly, severe hypoxemia was an independent predictor of NIV failure in hypercapnic patients of all origins, whereas altered consciousness at admission and ventilatory settings had no influence on outcome.

\section{REFERENCES}

1. Lightowler JV, Wedzicha JA, Elliott MW, Ram FS. Non-invasive positive pressure ventilation to treat respiratory failure resulting from exacerbations of chronic obstructive pulmonary disease: Cochrane systematic review and meta-analysis. BMJ 2003;326(7382):185.

2. Keenan SP, Sinuff T, Cook DJ, Hill NS. Which patients with acute exacerbation of chronic obstructive pulmonary disease benefit from noninvasive positive-pressure ventilation? A systematic review of the literature. Ann Intern Med 2003;138(11):861-870.

3. Masip J, Roque M, Sanchez B, Fernandez R, Subirana M, Exposito JA. Noninvasive ventilation in acute cardiogenic pulmonary edema: systematic review and meta-analysis. JAMA 2005;294(24):3124-3130.

4. Brochard L, Isabey D, Piquet J, Amaro P, Mancebo J, Messadi AA, et al. Reversal of acute exacerbations of chronic obstructive lung disease by inspiratory assistance with a face mask. N Engl J Med 1990;323(22):1523-1530.

5. Brochard L, Mancebo J, Wysocki M, Lofaso F, Conti G, Rauss A, et al. Noninvasive ventilation for acute exacerbations of chronic obstructive pulmonary disease. N Engl J Med 1995;333(13):817-822.

6. Chandra D, Stamm JA, Taylor B, Ramos RM, Satterwhite L, Krishnan JA, et al. Outcomes of noninvasive ventilation for acute exacerbations of chronic obstructive pulmonary disease in the United States, 1998-2008. Am J Respir Crit Care Med 2012;185(2): 152-159.

7. Esteban A, Ferguson ND, Meade MO, Frutos-Vivar F, Apezteguia $\mathrm{C}$, Brochard L, et al. Evolution of mechanical ventilation in re- 


\section{Noninvasive Ventilation for Acute Hypercapnic Respiratory Failure}

sponse to clinical research. Am J Respir Crit Care Med 2008;177(2): 170-177.

8. Demoule A, Girou E, Richard JC, Taille S, Brochard L. Increased use of noninvasive ventilation in French intensive care units. Intensive Care Med 2006;32(11):1747-1755.

9. Jolliet P, Tassaux D, Roeseler J, Burdet L, Broccard A, D'Hoore W, et al. Helium-oxygen versus air-oxygen noninvasive pressure support in decompensated chronic obstructive disease: a prospective, multicenter study. Crit Care Med 2003;31(3):878-884.

10. Maggiore SM, Richard JC, Abroug F, Diehl JL, Antonelli M, Sauder $\mathrm{P}$, et al. A multicenter, randomized trial of noninvasive ventilation with helium-oxygen mixture in exacerbations of chronic obstructive lung disease. Crit Care Med 2010;38(1):145-151.

11. Phua J, Kong K, Lee KH, Shen L, Lim TK. Noninvasive ventilation in hypercapnic acute respiratory failure due to chronic obstructive pulmonary disease vs other conditions: effectiveness and predictors of failure. Intensive Care Med 2005;31(4):533-539.

12. Schettino G, Altobelli N, Kacmarek RM. Noninvasive positivepressure ventilation in acute respiratory failure outside clinical trials: experience at the Massachusetts General Hospital. Crit Care Med 2008;36(2):441-447.

13. Ambrosino N, Foglio K, Rubini F, Clini E, Nava S, Vitacca M. Non-invasive mechanical ventilation in acute respiratory failure due to chronic obstructive pulmonary disease: correlates for success. Thorax 1995;50(7):755-757.

14. Anton A, Guell R, Gomez J, Serrano J, Castellano A, Carrasco JL, et al. Predicting the result of noninvasive ventilation in severe acute exacerbations of patients with chronic airflow limitation. Chest 2000; 117(3):828-833.

15. Confalonieri M, Garuti G, Cattaruzza MS, Osborn JF, Antonelli M, Conti $\mathrm{G}$, et al. A chart of failure risk for noninvasive ventilation in patients with COPD exacerbation. Eur Respir J 2005;25(2):348-355.

16. Contou D, Fragnoli C, Cordoba-Izquierdo A, Boissier F, BrunBuisson C, Thille AW. Predictive factors for NIV failure in acute hypercapnic respiratory failure. Intensive Care Med 2012;38. 25th annual meeting of the ESICM: abstract \#0253.

17. Ely EW, Truman B, Shintani A, Thomason JW, Wheeler AP, Gordon S, et al. Monitoring sedation status over time in ICU patients: reliability and validity of the Richmond Agitation-Sedation Scale (RASS). JAMA 2003;289(22):2983-2991.

18. Nouira S, Boukef R, Bouida W, Kerkeni W, Beltaief K, Boubaker H, et al. Non-invasive pressure support ventilation and CPAP in cardiogenic pulmonary edema: a multicenter randomized study in the emergency department. Intensive Care Med 2011;37(2):249-256.

19. Nava S, Carbone G, DiBattista N, Bellone A, Baiardi P, Cosentini R, et al. Noninvasive ventilation in cardiogenic pulmonary edema: a multicenter randomized trial. Am J Respir Crit Care Med 2003; 168(12):1432-1437.
20. Plant PK, Owen JL, Elliott MW. Early use of non-invasive ventilation for acute exacerbations of chronic obstructive pulmonary disease on general respiratory wards: a multicentre randomised controlled trial. Lancet 2000;355(9219):1931-1935.

21. Carrillo A, Ferrer M, Gonzalez-Diaz G, Lopez-Martinez A, Llamas $\mathrm{N}$, Alcazar M, et al. Non-invasive ventilation in acute hypercapnic respiratory failure due to obesity-hypoventilation syndrome and COPD. Am J Respir Crit Care Med 2012;186(12):1279-1785.

22. Duarte AG, Justino E, Bigler T, Grady J. Outcomes of morbidly obese patients requiring mechanical ventilation for acute respiratory failure. Crit Care Med 2007;35(3):732-737.

23. Piper AJ, Sullivan CE. Effects of short-term NIPPV in the treatment of patients with severe obstructive sleep apnea and hypercapnia. Chest 1994;105(2):434-440.

24. Phua J, Ang YL, See KC, Mukhopadhyay A, Santiago EA, Dela Pena EG, et al. Noninvasive and invasive ventilation in acute respiratory failure associated with bronchiectasis. Intensive Care Med 2010;36(4):638-647.

25. Robino C, Faisy C, Diehl JL, Rezgui N, Labrousse J, Guerot E. Effectiveness of non-invasive positive pressure ventilation differs between decompensated chronic restrictive and obstructive pulmonary disease patients. Intensive Care Med 2003;29(4):603-610.

26. Seneviratne J, Mandrekar J, Wijdicks EF, Rabinstein AA. Noninvasive ventilation in myasthenic crisis. Arch Neurol 2008;65(1):54-58.

27. Bach JR, Ishikawa Y, Kim H. Prevention of pulmonary morbidity for patients with Duchenne muscular dystrophy. Chest 1997;112(4): 1024-1028.

28. Demoule A, Girou E, Richard JC, Taillé S, Brochard L. Benefits and risks of success or failure of noninvasive ventilation. Intensive Care Med 2006;32(11):1756-1765.

29. Nava S, Navalesi P, Conti G. Time of non-invasive ventilation. Intensive Care Med 2006;32(3):361-370.

30. Diaz GG, Alcaraz AC, Talavera JC, Perez PJ, Rodriguez AE, Cordoba FG, et al. Noninvasive positive-pressure ventilation to treat hypercapnic coma secondary to respiratory failure. Chest $2005 ; 127(3)$ : 952-960.

31. Scala R, Naldi M, Archinucci I, Coniglio G, Nava S. Noninvasive positive pressure ventilation in patients with acute exacerbations of COPD and varying levels of consciousness. Chest 2005;128(3): $1657-1666$

32. Ely EW, Baker AM, Dunagan DP, Burke HL, Smith AC, Kelly PT, et al. Effect on the duration of mechanical ventilation of identifying patients capable of breathing spontaneously. N Engl J Med 1996; 335(25):1864-1869.

33. Girard TD, Kress JP, Fuchs BD, Thomason JW, Schweickert WD, Pun BT, et al. Efficacy and safety of a paired sedation and ventilator weaning protocol for mechanically ventilated patients in intensive care (Awakening and Breathing Controlled trial): a randomised controlled trial. Lancet 2008;371(9607):126-134.

This article is approved for Continuing Respiratory Care Education credit. For information and to obtain your CRCE

(free to AARC members) visit www.rcjournal.com

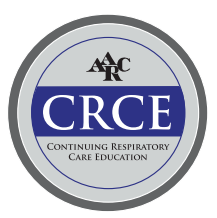

